\title{
Advanced in-situ electron-beam lithography for deterministic nanophotonic device processing
}

Cite as: Rev. Sci. Instrum. 86, 073903 (2015); https://doi.org/10.1063/1.4926995

Submitted: 22 May 2015. Accepted: 07 July 2015 . Published Online: 21 July 2015

Arsenty Kaganskiy, Manuel Gschrey, Alexander Schlehahn, Ronny Schmidt, Jan-Hindrik Schulze,

Tobias Heindel (D), André Strittmatter (D), Sven Rodt, and Stephan Reitzenstein (iD)

\section{ARTICLES YOU MAY BE INTERESTED IN}

In situ electron-beam lithography of deterministic single-quantum-dot mesa-structures using low-temperature cathodoluminescence spectroscopy

Applied Physics Letters 102, 251113 (2013); https://doi.org/10.1063/1.4812343

Resolution and alignment accuracy of low-temperature in situ electron beam lithography for nanophotonic device fabrication

Journal of Vacuum Science \& Technology B 33, 021603 (2015); https://

doi.org/10.1116/1.4914914

Enhanced photon-extraction efficiency from InGaAs/GaAs quantum dots in deterministic photonic structures at $1.3 \mu \mathrm{m}$ fabricated by in-situ electron-beam lithography

AIP Advances 8, 085205 (2018); https://doi.org/10.1063/1.5038137

\section{Lock-in Amplifiers up to $600 \mathrm{MHz}$}
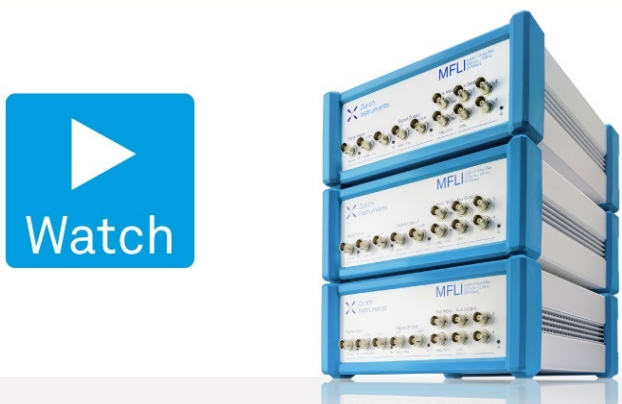

Rev. Sci. Instrum. 86, 073903 (2015); https://doi.org/10.1063/1.4926995 


\title{
Advanced in-situ electron-beam lithography for deterministic nanophotonic device processing
}

\author{
Arsenty Kaganskiy, ${ }^{1}$ Manuel Gschrey, ${ }^{1}$ Alexander Schlehahn, ${ }^{1}$ Ronny Schmidt, ${ }^{1}$ \\ Jan-Hindrik Schulze, ${ }^{1}$ Tobias Heindel, ${ }^{1}$ André Strittmatter, ${ }^{1,2}$ Sven Rodt, ${ }^{1, a)}$ \\ and Stephan Reitzenstein ${ }^{1}$ \\ ${ }^{1}$ Institut für Festkörperphysik, Technische Universität Berlin, Hardenbergstraße 36, D-10623 Berlin, Germany \\ ${ }^{2}$ Otto-von-Guericke Universität Magdeburg, Universitätsplatz, 2, D-39106 Magdeburg, Germany
}

(Received 22 May 2015; accepted 7 July 2015; published online 21 July 2015)

\begin{abstract}
We report on an advanced in-situ electron-beam lithography technique based on high-resolution cathodoluminescence (CL) spectroscopy at low temperatures. The technique has been developed for the deterministic fabrication and quantitative evaluation of nanophotonic structures. It is of particular interest for the realization and optimization of non-classical light sources which require the pre-selection of single quantum dots (QDs) with very specific emission features. The two-step electron-beam lithography process comprises (a) the detailed optical study and selection of target QDs by means of CL-spectroscopy and (b) the precise retrieval of the locations and integration of target QDs into lithographically defined nanostructures. Our technology platform allows for a detailed pre-process determination of important optical and quantum optical properties of the QDs, such as the emission energies of excitonic complexes, the excitonic fine-structure splitting, the carrier dynamics, and the quantum nature of emission. In addition, it enables a direct and precise comparison of the optical properties of a single QD before and after integration which is very beneficial for the quantitative evaluation of cavity-enhanced quantum devices. ( 2015 AIP Publishing LLC. [http://dx.doi.org/10.1063/1.4926995]
\end{abstract}

\section{INTRODUCTION}

Future applications of nanophotonic devices in quantum information technology ${ }^{1}$ set stringent requirements on their optical properties. Key components are non-classical light sources $^{2,3}$ which need to emit single photons on demand with high extraction efficiency, ${ }^{4,5}$ high suppression of multiphoton emission, ${ }^{4}$ and high indistinguishability ${ }^{5,6}$ to mention only the most important requirements. Moreover, when going beyond simple proof-of-concept demonstrations towards the implementation of more complex scenarios such as quantum networks, ${ }^{7}$ it is vital to develop also deterministic nanofabrication platforms with reasonably high process yield. At the same time also techniques for a better quantitative evaluation of photonic quantum devices and their processing need to be established. While numerical simulations can provide a basic understanding of the functional principles of nanostructured devices, ${ }^{8,9}$ there is a need for advanced experimental techniques that trace their influence in real structures within a one-to-one comparison of the optical properties before and after processing for a quantitative evaluation with high accuracy. A modification of the spontaneous decay rate via the Purcell effect by spectral matching to a resonator structure $^{10,11}$ is a prominent example, which should be monitored at the single emitter level. Another important property is the exciton's fine-structure splitting (FSS) in quantum dots $(\mathrm{QDs})^{12}$. A controlled integration of single QDs with vanishing FSS into nanophotonic structures will enable the realization of polarization-entangled-photon-pair emitters. ${ }^{13}$ The basic

a)srodt@physik.tu-berlin.de requirement for a selective QD choice and a direct comparison is a deterministic fabrication process that enables the integration of a pre-selected QD into a high-quality nanophotonic structure. While a number of deterministic approaches exists, ${ }^{14-18}$ a fabrication technique based on (a) electron-beam lithography together with (b) a high positioning accuracy and (c) detailed spectroscopic methods is desirable to enable a flexible and adapted device design. Cathodoluminescence lithography (CLL) already provides (a) and (b), and partly (c), as the investigation can only be accomplished in a very limited amount of time, restricted by the resist properties. ${ }^{19}$ To overcome this difficulty, we introduce in the present work a fabrication scheme that extends the basic CLL process with a resist-free and marker-based pre-characterization step, allowing for a comprehensive investigation of the QDs prior to device processing. To demonstrate this enhanced cathodoluminescence lithography (eCLL) technique, we isolate precharacterized QDs and integrate them into mesa structures for an exemplarily follow-up comparison with the initial results. We trace the emission energies as well as the FSS of the single QDs, both being crucial parameters, e.g., for resonance matching in cavity structures and for the generation of entangled photon-pairs, respectively. Moreover, in-situ measurements of the luminescence decay and the second-order photon-autocorrelation function demonstrate the extensive spectroscopic capabilities of eCLL.

\section{EXPERIMENTAL AND RESULTS}

The advanced in-situ electron-beam lithography presented in this work is based on our CLL platform reported 
previously. ${ }^{20}$ In CLL, a quick CL mapping is applied for the pre-registering of single QDs with selected emission energies. Up to now, the dwell times for spectroscopic investigations at each mapping point on the sample were restricted by the sensitivity of the electron-beam-sensitive resist that is present on the sample during the full CLL process. Typical dwell times were in the range of $50 \mathrm{~ms}$ to $100 \mathrm{~ms}$, depending on the chosen acceleration voltage of the primary electrons. These dwell times are significantly lower than typical integration times of up to several 10 min required for a detailed spectroscopic precharacterization of QDs. In order to overcome the limitation of CLL in terms of small dwell times in the selection process, we introduce an advanced in-situ electron-beam lithography technology platform which also includes a comprehensive pre-characterization of the sample to satisfy the demands of selecting emitters with very specific and stringent spectral specifications. The pre-process CL characterization includes, e.g., measurements as a function of the excitation density, the determination of the luminescence's polarization, timeresolved experiments, as well as investigations of the quantum nature of emission by Hanbury-Brown and Twiss experiments. Our enhanced CLL process relies on macroscopic marker structures on the sample's surface which are patterned by a standard UV lithography step and a subsequent lift-off process. Utilization of marker structures was also reported by Nogues et al. ${ }^{15}$ who applied CL to determine QD positions with respect to the markers. Afterwards, they performed EBL with a sole reference to the markers in secondary-electron images where they obtain a sum of errors from the first localization, the ebeam realignment, and the second localization. Kojima et al. ${ }^{16}$ used pre-fabricated marker structures in combination with micro-photoluminescence spectroscopy ( $\mu \mathrm{PL}$ ) to pin the QDs' positions and performed EBL with respect to the markers afterwards. This technique has two drawbacks, as $\mu \mathrm{PL}$ has a reduced lateral resolution as compared to $\mathrm{CL}$ and they also have to rely on an as-good-as-possible second localization with respect to the markers. We bypass such marker-based localization errors as our marker structures only serve for a rough re-positioning on the sample while the exact positions of the QDs are determined by the high-resolution CL mapping and accurate alignment within the CLL work flow as described below.

The process flow of eCLL is sketched in Fig. 1. First, alignment markers are processed on the sample by standard UV lithography before it is mapped and pre-characterized by high-resolution CL spectroscopy—including all envisaged spectroscopic measurements (Fig. 1(a)). Next, the sample is spin-coated with an electron-beam sensitive resist and CLL is performed on the pre-characterized areas (Fig. 1(b)). As a key feature of our scheme, the second determination of QD positions is solely based on the high-resolution CL mapping with a lateral accuracy of $25 \mathrm{~nm}^{21}$ on the spin-coated sample. The subsequent EBL step at cryogenic temperature is performed in immediate succession, to avoid lateral misalignment caused by a possible temporal drift of the sample. After development of the resist and etching of the sample, the single encapsulated QDs are post-characterized (Fig. 1(c)). This procedure enables a precise one-to-one comparison of the opto-electronic features of single quantum emitters before and after processing

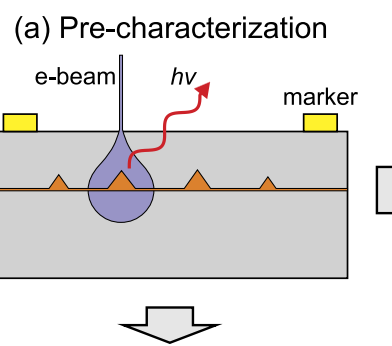

(d) Comparison

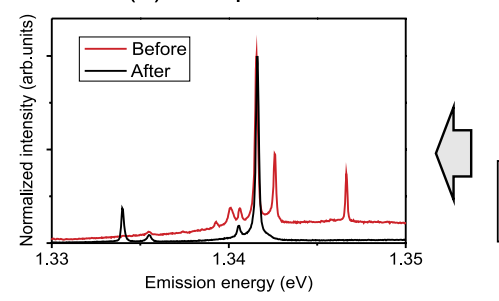

FIG. 1. Schematics of the eCLL process with the use of CL for optical characterization. First, marker structures are applied to the sample and it is pre-characterized (a). Then, the CLL process is conducted and the single QDs are incorporated into nanophotonic structures (b). Post-characterization of the processed structures (c) allows for a direct comparison (d) with the results from (a)

(Fig. 1(d)). Thanks to the macroscopic marker structures, $\mu \mathrm{PL}$ is also applicable in the pre- and post-characterization steps.

The eCLL work flow combines the great advantages of extensive pre-characterization of single QDs by a manifold of spectroscopic experiments with the high lateral accuracy of CLL-sample-processing. The overall lateral accuracy of the full CLL process was determined to be $34 \mathrm{~nm} .{ }^{21}$ It could be improved by utilizing a cryostat that exhibits almost no thermal drift. Target nanophotonic application might be the integration of QDs with a vanishing fine-structure splitting and matching emission energy in microlens or microcavity structures for the emission of polarization-entangled photon pairs from the biexciton-exciton radiative cascade ${ }^{12}$ or the fabrication of single-photon sources with identical emission for the generation of indistinguishable photons in quantum communication schemes. ${ }^{7}$

The sample under investigation was grown by metalorganic chemical vapor deposition (MOCVD) on GaAs(001) substrate and includes a lower distributed Bragg reflector (DBR) for an enhanced photon-extraction efficiency of the QD luminescence. First, $300 \mathrm{~nm}$ of GaAs was deposited followed by the DBR consisting of 23 pairs of $\lambda / 4$-layers of $77 \mathrm{~nm}$ $\mathrm{Al}_{0.9} \mathrm{Ga}_{0.1} \mathrm{As}$ and $65.7 \mathrm{~nm}$ GaAs. The targeted central wavelength of the DBR's stopband is $\lambda=935 \mathrm{~nm}$. Next, $65.7 \mathrm{~nm}$ of GaAs was deposited, followed by self-organized InGaAs QDs in the Stranski-Krastanow growth mode at a temperature of $500{ }^{\circ} \mathrm{C}$ during a growth interruption of $35 \mathrm{~s}$. Finally, the QDs were capped by $400 \mathrm{~nm}$ of GaAs. The GaAs capping layer provides the material for processing the CLL structures. The QD density was estimated to be $\approx 10^{7} \mathrm{~cm}^{-2}$.

Alignment markers were patterned on the sample's surface by a standard UV lithography step and a successive liftoff process. Figure 2(c) shows a section of such a marker structure that consists of gold markers including boundarymarks and numbered labels for every $50 \mu \mathrm{m} \times 50 \mu \mathrm{m}$ field. The numbering allows for an easy relocation of a given field. 


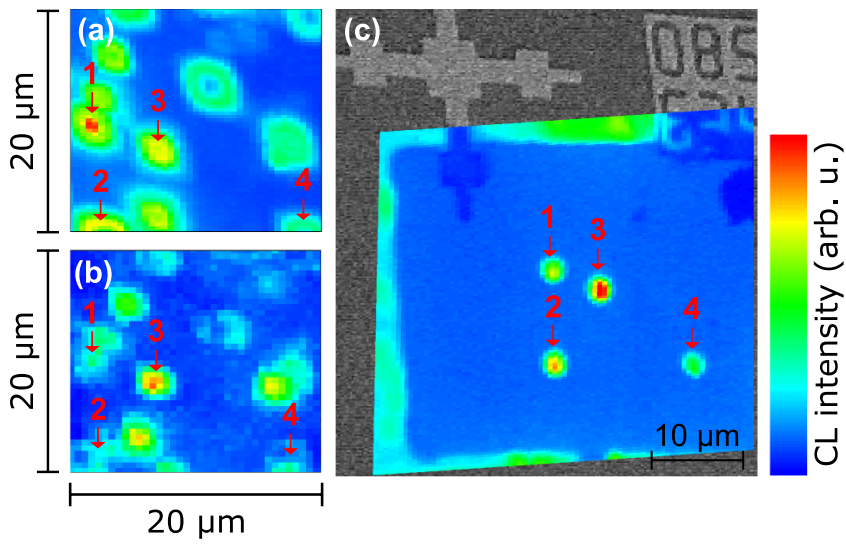

FIG. 2. CL maps for the three stages of the enhanced CLL process. (a) CL map with longer dwell time (100 ms) per map pixel during the initial pre-characterization. The luminescence spots from the selected QDs are marked by red arrows and numbers for easier tracking. (b) CL map as taken on the resist-coated sample to relocate the pre-selected QDs with dwell times per pixel of $30 \mathrm{~ms}$. (c) CL map of the fully processed structure in front of a larger area secondary-electron image. The macroscopic markers used for relocating the $50 \mu \mathrm{m} \times 50 \mu \mathrm{m}$ fields are visible in the top region. All maps show emission within an energy range from $1.337 \mathrm{eV}$ to $1.345 \mathrm{eV}$.

Next, a number of single QDs in different fields are investigated by $\mathrm{CL}$ at a temperature of $5 \mathrm{~K}$. The emission lines from excitonic and biexcitonic recombinations are identified by performing excitation- and polarization-dependent measurements. Special emphasis is put on the FSS of the excitonic emission. To demonstrate the versatility of our CLL setup, we additionally performed exemplarily time-resolved $\mathrm{CL}$ experiments. Time-resolved single-photon counting was used for determination of CL decay times and CL photon autocorrelation to determine the degree of single-photon emission. Additionally, a CL map is taken (Fig. 2(a)) to facilitate the relocation process of the QDs.

The in-situ electron-beam-lithography process is initiated by spin-coating a $225 \mathrm{~nm}$ thick polymethyl-methacrylate (PMMA) layer on the sample surface. Then, the sample is remounted in the CLL setup and cooled down to a temperature of $5 \mathrm{~K}$. The pre-characterized QDs in the numbered fields are easily relocated by roughly accessing the desired area and by fine-tuning the position by taking single-shot secondaryelectron images. The incorporated electron dose per image of $0.7 \mu \mathrm{C} / \mathrm{cm}^{2}$ is so low that the PMMA resist is almost not affected. When the target write-field is reached, the actual CLL process starts. The sample area is mapped by CL with short integration times ( $\approx 30 \mathrm{~ms}$ ) per pixel (Fig. 2(b)), introducing a dose of $6.5 \mathrm{mC} / \mathrm{cm}^{2}$. During this mapping, the PMMA chains are cracked and the resist becomes soluble for the later development step. Within this map, the pre-characterized QDs are easily identified and precisely relocated (cf. Figs. 2(a) and 2(b)). Now, circular disk patterns with diameters of $2 \mu \mathrm{m}$ are written into the resist by applying an electron dose of $14 \mathrm{mC} / \mathrm{cm}^{2}$. This large electron dose leads to a cross-linking and carbonization of the afore cracked PMMA chains and it becomes resistant against the developer. When the CLL process is finished on all QDs, the sample is transferred out of the CL-system and it is developed in a mixture of methylisobutylketon (MIBK) and isopropyl alcohol (IPA) at room temperature. Next, dry etching is performed in an inductively coupled-plasma reactive-ion etching (ICP-RIE) plasma
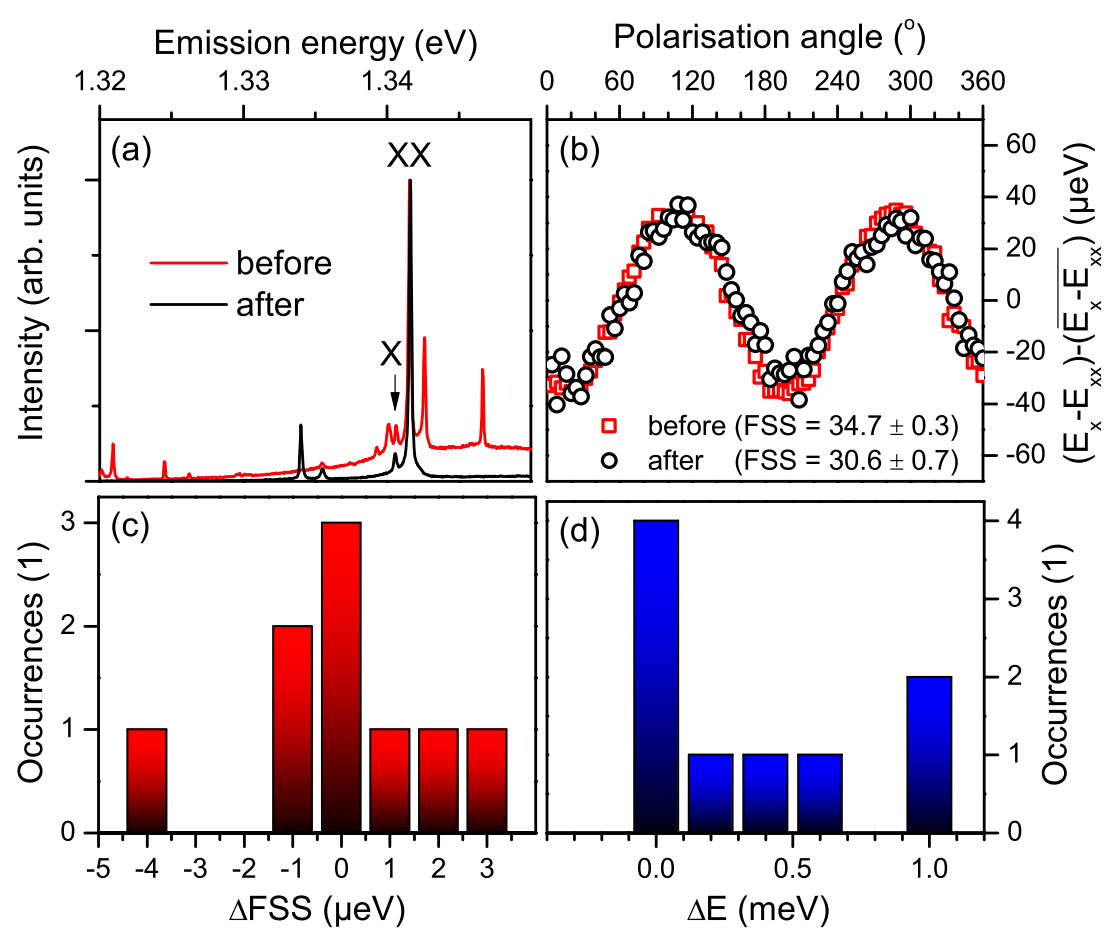

FIG. 3. Exemplary spectra (a) and polarization-resolved differential shifts of exciton and biexciton emissions (b) for a single QD before and after processing of the mesa structure. For (b), the polarization dependent emission energies of exciton and biexciton were extracted and the differential shift was calculated as given by the $y$-axis title of (b). This procedure eliminates a possible collective shift of the spectra during the polarization scan and reduces the overall statistical error. The fine-structure splitting is given by half of the amplitude. Extracted variations of the fine-structure splitting $(\overline{\Delta F S S}=0 \mu \mathrm{eV}, \sigma=2 \mu \mathrm{eV})(\mathrm{c})$ and of the exciton's recombination energy $(\overline{\Delta E}=0.4 \mathrm{meV}, \sigma=0.4 \mathrm{meV})(\mathrm{d})$ for nine QDs. Energy values from the pre-characterization step were subtracted from those of the mesa structures. 

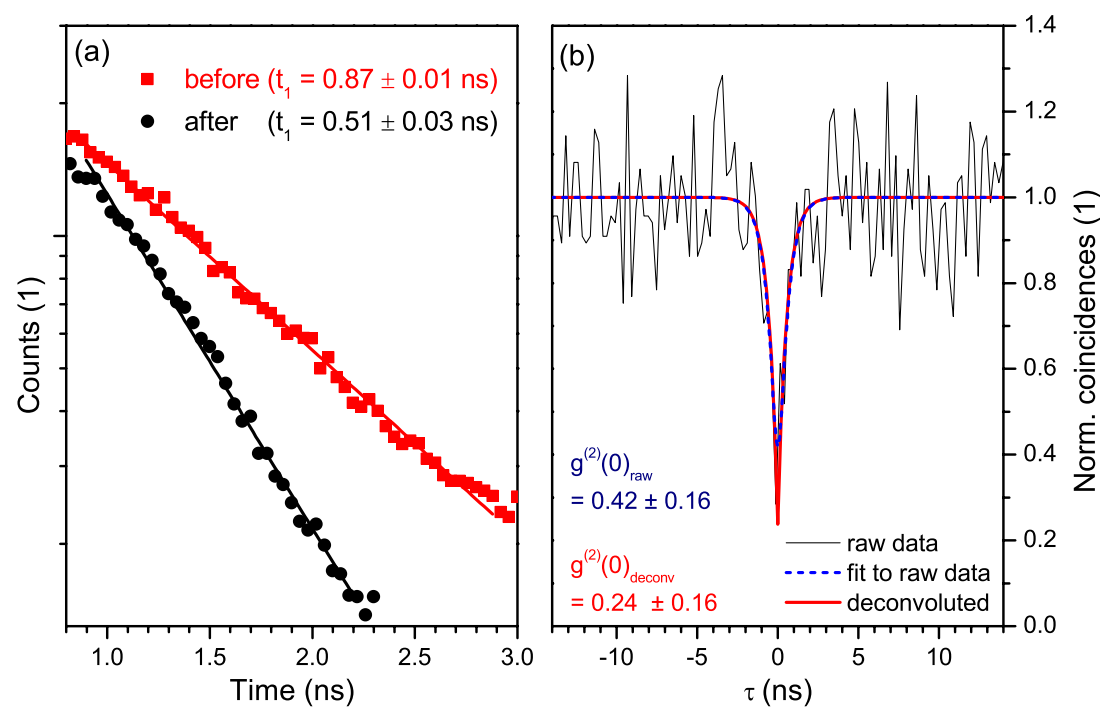

FIG. 4. Time-resolved CL measurements. (a) Decay of an uncharged biexciton state before and after processing of the mesa structure. Solid lines represent exponential fits to the data. The QD in the mesa exhibits a reduced decay time by a factor of $1.7 \pm 0.1$. (b) Auto-correlation data $g^{(2)}(\tau)$ of the transition of a positively charged exciton. The corrected $g_{\text {deconv }}^{(2)}(0)$ value of $0.24 \pm 0.16$ clearly demonstrates emission of single photons.

under a pressure of $0.08 \mathrm{~Pa}$ with $100 \mathrm{~W}$ ICP coil power and $-213 \mathrm{~V}$ substrate bias voltage. A combination of $\mathrm{Cl}_{2}: \mathrm{BCl}_{3}: \mathrm{Ar}$, with a ratio of 1.3:4.3:1.1, is used to reach a selectivity of GaAs against unexposed PMMA of 2. Realizing an etch depth of up to $430 \mathrm{~nm}$, the disk profiles are transferred from the inverted PMMA into the semiconductor and the QD layer around the resulting mesa structures is removed.

After the processing, the single QDs in their mesa structures are investigated in the same way as in the pre-characterization step. As an example, Fig. 3 displays spectra and polarization-resolved differential shifts of the exciton's and biexciton's emission energy for one and the same QD before and after fabrication of the mesa. It is clearly demonstrated in (a) that only a single QD remained in the mesa with the same emission energies of its excitonic complexes as before. A total of nine QDs were investigated in this experiment. Figure 3 displays the relative changes in the exciton's FSS (c) and emission energy (d) (values obtained for the mesa structures minus the respective energies before processing of the mesas). The energetic values can be determined with high accuracy and are almost unaffected by the patterning of the sample: there is a slight increase in emission energy $(\overline{\Delta E}=0.4 \mathrm{meV}$, $\sigma=0.4 \mathrm{meV})$, while the change of FSS $(\overline{\Delta F S S}=0 \mu \mathrm{eV}$, $\sigma=2 \mu \mathrm{eV}$ ) lies within the statistical error. Here, the change of emission energy is most probably related to a different electrostatic environment of the QD in the microstructure as compared to the planar sample. At this point, a more thorough analysis of the underlying mechanisms is not possible, as the QD's charge environment on a microscopic scale is unknown. We would like to note that the slight shift of the emission energy can easily be compensated by temperature tuning in the case of microcavity structures ${ }^{22}$ or in quantum-repeater scenarios ${ }^{23}$ where a spectral matching is vital.

Fig. 4 shows exemplary time-resolved CL experiments. In (a), results for the single-photon counting are displayed for an accumulation time of $25 \mathrm{~min}$. The decay time is shortened from $(0.87 \pm 0.01) \mathrm{ns}$ to $(0.51 \pm 0.03) \mathrm{ns}$. The shortening of the
CL-decay time indicates a slight Purcell factor of $1.7 \pm 0.1$ due to a larger local density of optical states in the presence of the mesa structure. In principle, the reduced CL decay time could also be attributed to additional nonradiative recombination centers at the etched microlens surface. However, since the QD is still surrounded by at least $400 \mathrm{~nm}$ of GaAs in vertical direction and $1000 \mathrm{~nm}$ in lateral direction, we do not expect a significant influence of such centers on the decay dynamics of the QD. ${ }^{24,25}$ Fig. 4(b) shows the ability of our CLL setup to also perform auto-correlation measurements. Such measurements are crucial to, e.g., ensure the single-photon emission-character of a selected emitter. The $g^{(2)}(\tau)$ statistics was recorded for $3.5 \mathrm{~h}$. The black line gives the raw data and the red line is a fit where the finite time-resolution of the APDs was considered by deconvolution. ${ }^{26}$ The corrected $g^{(2)}(0)$ value of $0.24 \pm 0.16$ clearly indicates the emission of single photons.

\section{SUMMARY}

In summary, we have developed a powerful in-situ electron-beam-lithography technology platform that allows for a thorough and comprehensive pre-characterization of single QDs before they are deterministically incorporated into etched nanophotonic structures. By fabricating mesa structures with pre-selected single QDs inside, we exemplarily demonstrated the feasibility of our approach. We also compared the QDs' emission energies and fine-structure splittings before and after fabrication of the mesas to evaluate its influence on the electronic properties of the QDs. Only a slight increase of the emission energies was found that can easily be compensated by temperature tuning, while no significant change of the FSS could be detected. Time-resolved measurements indicate cavity effects in the mesa structures resulting in a twofold shortening of the decay time by the Purcell effect. As such, eCLL has proven to be a powerful tool for the advanced and deterministic fabrication of nanophotonic 
structures and will be very beneficial for the optimization and fabrication of tailored quantum light sources in the future.

\section{ACKNOWLEDGMENTS}

We acknowledge support from Deutsche Forschungsgemeinschaft (DFG) through SFB 787 "Semiconductor Nanophotonics: Materials, Models, Devices."

${ }^{1}$ N. Gisin and R. Thew, Nat. Photonics 1, 165 (2007).

${ }^{2}$ J. Kim, O. Benson, H. Kan, and Y. Yamamoto, Nature 397, 500 (1999).

${ }^{3}$ P. Michler, Science 290, 2282 (2000).

${ }^{4}$ J. Claudon, J. Bleuse, N. S. Malik, M. Bazin, P. Jaffrennou, N. Gregersen, C. Sauvan, P. Lalanne, and J.-M. Gérard, Nat. Photonics 4, 174 (2010).

${ }^{5}$ O. Gazzano, S. Michaelis de Vasconcellos, C. Arnold, A. Nowak, E. Galopin, I. Sagnes, L. Lanco, A. Lemaître, and P. Senellart, Nat. Commun. 4, 1425 (2013).

${ }^{6}$ Y.-J. Wei, Y.-M. He, M.-C. Chen, Y.-N. Hu, Y. He, D. Wu, C. Schneider, M. Kamp, S. Höfling, C.-Y. Lu, and J.-W. Pan, Nano Lett. 14, 6515 (2014).

${ }^{7}$ J. L. O’Brien, A. Furusawa, and J. Vučković, Nat. Photonics 3, 687 (2009).

${ }^{8}$ J. Vučković, M. Pelton, A. Scherer, and Y. Yamamoto, Phys. Rev. A 66 , 023808 (2002).

${ }^{9}$ F. Ding, T. Stöferle, L. Mai, A. Knoll, and R. F. Mahrt, Phys. Rev. B 87, 161116(R) (2013).

${ }^{10}$ J. Gérard, B. Sermage, B. Gayral, B. Legrand, E. Costard, and V. ThierryMieg, Phys. Rev. Lett. 81, 1110 (1998).

${ }^{11}$ G. Solomon, M. Pelton, and Y. Yamamoto, Phys. Rev. Lett. 86, 3903 (2001).

${ }^{12}$ O. Benson, C. Santori, M. Pelton, and Y. Yamamoto, Phys. Rev. Lett. 84 2513 (2000).
${ }^{13}$ G. Juska, V. Dimastrodonato, L. O. Mereni, A. Gocalinska, and E. Pelucchi, Nat. Photonics 7, 527 (2013).

${ }^{14}$ A. Dousse, L. Lanco, J. Suffczyński, E. Semenova, A. Miard, A. Lemaître, I. Sagnes, C. Roblin, J. Bloch, and P. Senellart, Phys. Rev. Lett. 101, 267404 (2008).

${ }^{15}$ G. Nogues, Q. Merotto, G. Bachelier, E. Hye Lee, and J. Dong Song, Appl. Phys. Lett. 102, 231112 (2013).

${ }^{16}$ T. Kojima, K. Kojima, T. Asano, and S. Noda, Appl. Phys. Lett. 102, 011110 (2013).

${ }^{17}$ A. K. Nowak, S. L. Portalupi, V. Giesz, O. Gazzano, C. Dal Savio, P.-F. Braun, K. Karrai, C. Arnold, L. Lanco, I. Sagnes et al., Nat. Commun. 5, 3240 (2014).

${ }^{18}$ M. Pfeiffer, K. Lindfors, H. Zhang, B. Fenk, F. Phillipp, P. Atkinson, A. Rastelli, O. G. Schmidt, H. Giessen, and M. Lippitz, Nano Lett. 14, 197 (2014).

${ }^{19}$ M. Gschrey, R. Schmidt, A. Kaganskiy, S. Rodt, and S. Reitzenstein, J. Vac. Sci. Technol., B 32, 061601 (2014).

${ }^{20}$ M. Gschrey, F. Gericke, A. Schüßler, R. Schmidt, J.-H. Schulze, T. Heindel, S. Rodt, A. Strittmatter, and S. Reitzenstein, Appl. Phys. Lett. 102, 251113 (2013).

${ }^{21}$ M. Gschrey, R. Schmidt, J.-H. Schulze, A. Strittmatter, S. Rodt, and S. Reitzenstein, J. Vac. Sci. Technol., B 33, 021603 (2015).

${ }^{22}$ T. Heindel, C. Schneider, M. Lermer, S. H. Kwon, T. Braun, S. Reitzenstein, S. Höfling, M. Kamp, and A. Forchel, Appl. Phys. Lett. 96, 011107 (2010).

${ }^{23}$ P. Gold, A. Thoma, S. Maier, S. Reitzenstein, C. Schneider, S. Höfling, and M. Kamp, Phys. Rev. B 89, 035313 (2014).

${ }^{24}$ C. F. Wang, A. Badolato, I. Wilson-Rae, P. M. Petroff, E. Hu, J. Urayama, and A. Imamoğlu, Appl. Phys. Lett. 85, 3423 (2004).

${ }^{25}$ J. Johansen, S. Stobbe, I. S. Nikolaev, T. Lund-Hansen, P. T. Kristensen, J. M. Hvam, W. L. Vos, and P. Lodahl, Phys. Rev. B 77, 073303 (2008).

${ }^{26}$ P. Michler, A. Imamoğlu, M. D. Mason, P. J. Carson, G. F. Strouse, and S. K. Buratto, Nature 406, 968 (2000). 